\title{
Two-photon-induced Microfabrication of Flexible Optical Waveguides
}

\author{
Josef KUMPFMUELLER ${ }^{* 1}$, Klaus STADLMANN ${ }^{* 2}$, Valentin SATZINGER ${ }^{* 3}{\text { Zhiquan } \text { LI }^{* 1} \text {, Juergen STAMPFL }}^{* 2}$ \\ and Robert LISKA ${ }^{* 1}$ \\ ${ }^{* 1}$ Institute of Applied Synthetic Chemistry, Vienna University of Technology, Lehargasse 2-6, \\ A-1060- Vienna, Austria \\ E-mail: josef.kumpfmueller@ias.tuwien.ac.at \\ ${ }^{*}$ Institute of Material Science and Technology, Vienna University of Technology, Favoritenstras- \\ se 9-11, A-1040-Vienna, Austria \\ ${ }^{*}$ MATERIALS-Institute for Surface Technology and Photonics, Joanneum Research, \\ Franz-Pichler-Strasse 30, A-8160-Weiz, Austria
}

\begin{abstract}
The Two-photon induced polymerization (2PP) is a novel concept in photopolymer chemistry allowing for elegant 3D direct laser writing (DLW) with resolutions down to several tens of nanometers. It has attracted significant attention in fields such as micro-electromechanical systems (MEMS), photonic crystals or surface plasmon polaritons. Additionally, 2PP can be applied for the structuring of optical waveguides by inducing a local refractive index increase over the surrounding cladding material. Herein, we present our latest work regarding high performance waveguides on the basis of polysiloxane/photopolymer hybrid materials, which we consider to be useful in the communication technology.

High refractive acrylic formulations were cured via 2PP in polysiloxane films. In comparison to former works the procedure could be significantly simplified by using a thixotropic, monomer-containing polysiloxane matrix formulation, which could be UV-cured after DLW. The enrichment of monomer during the DLW process in the illuminated regions enabled us to create a refractive index increase over the surrounding material and thus eliminating the impractical evaporating of the excess monomer by simply UV-curing the whole sample after 2PP-structuring. DLW of waveguide structures was carried out using our novel two-photon initiator B3FL.
\end{abstract}

DOI:10.2961/jlmn.2011.03.0004

Keywords: two-photon induced polymerization, waveguides, polysiloxane, thixotropic, refractive index increase

\section{Introduction}

When it comes to 3D solid freeform fabrication, 2PP is a very promising technique.[1] This emerging process has allowed researchers to achieve feature sizes down to a few tenths of nanometers in photosensitive resins. Optical data storage, microfluidics as well as biomedical applications are on the list of potential applications to name only a few. The fact, that polymerization only takes place within the tiny focal volume of the femtosecond laser awards 2PP with extra versatility compared to conventional lithographic techniques as for example microsterolithography and allows to tackle sophisticated problems such as connecting optical devices on optical printed circuit boards with waveguides. A 2PP induced refractive index change of in the range of 0.01 for $\Delta \mathrm{n} / \mathrm{n}$ enables efficient waveguiding.

A key issue in the latter application is to develop high performance materials, which locally undergo a refractive index change during two-photon illumination. Literature contains several examples of waveguide fabrication via 2PP: Direct writing within special glasses [2], selective organic crosslinking within Organic Modified Ceramics (ORMOCER)[3] materials and other sol-gel based materials [4] are typical examples. In an elegant work of Ishihara, the waveguide core is compiled via selective $2 \mathrm{PP}$ curing of radical monomers in a viscous mixture with cationically curable monomers, which are subsequently UV-cured to form the cladding.[5] Waveguides have also been fabricated by numerous other techniques such as hot embossing or reactive ion etching.[6]

An interesting niche application in the field of waveguiding are flexible printed circuit boards. For this purpose polydimethylsiloxane (PDMS) based materials are well suited due to their low optical damping behavior, excellent optical properties, high thermal stability, high flexibility and low cost. To our best knowledge, the pioneer work of fabricating PDMS-based waveguides via $2 \mathrm{PP}$ has been conducted by our group:[7] A casted PDMS film were cured via a Pt-catalyzed thermal hydrosilylation process followed by swelling the PDMS rubber with a photoreactive, acrylate based resin. Subsequently 2PP was carried out in the swollen film and finally the non-cured monomer was removed under vacuum and heat. Later on we developed a method, where the cladding material is cured leaving the photoreactive formulation untouched and saving the swelling step. A similar process using methacrylic monomers has been presented by Bichler et. al.[8]

In this work we want to present a new concept for the 2PP microfabrication of polysiloxane based flexible waveguides. Waveguides were written into a thixotropic film consisting of high refractive monomer formulation, lower refractive polyether containing photoreactive PDMS acrylate formulation and rheology additive. During the $2 \mathrm{PP}$ process higher refractive acrylate monomers are enriched in the illuminated area and cured. In the last step the thixotropic liquid is simply UV-cured. 
In contrast to the above mentioned approaches for flexible waveguides, our technology does not require orthogonal reactivities of higher and lower refractive material and the swelling as well as the evaporating step are eliminated. Fig. 1 gives an overview of the manufacturing process.
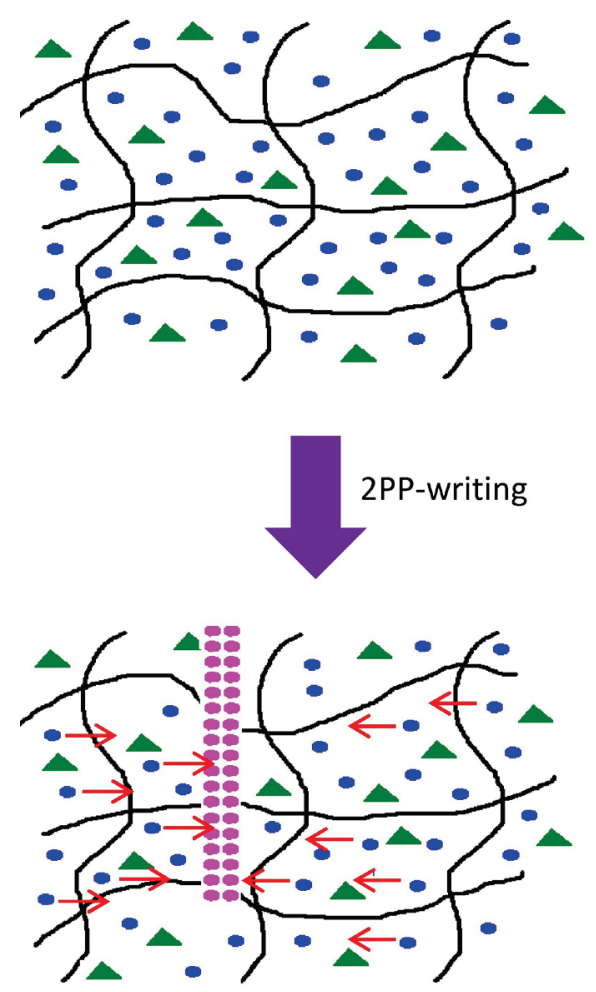

UV-curing

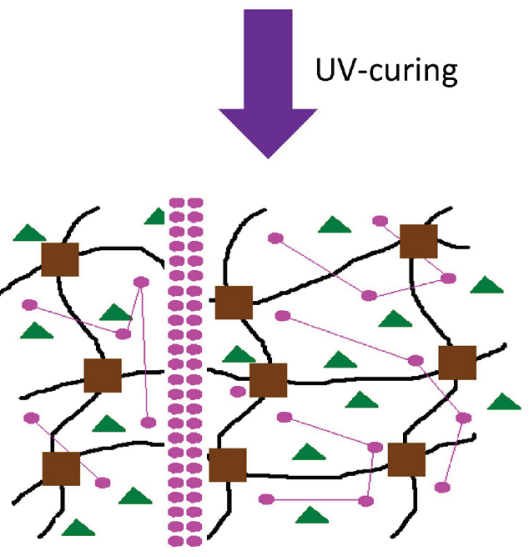

Fig. 1: Manufacturing process of flexible waveguides. $\Delta$ (rheology additive); - (Monomer); black wiggly line (polysiloxane matrix material); $\bullet$ (waveguide); $\bullet$, interconnected by lines (UV-cured monomer); " (polysiloxane matrix crosslink)

\section{Materials and Methods}

\subsection{Formulation}

Our novel two-photon-initiator B3FL [9] (Fig. 3) was dissolved in trimethylol propane triacrylate (TTA, Photomer) to a concentration of 0.1 weight $\%$ with respect to B3FL. This photoreactive monomer formulation was added to a mixture of BYK E 410 (BYK) and silicone polyether acrylate resin TEGO RAD 2200N (Evonik) and the onephoton-initiator Darocur 1173 (Ciba). The exact formulation is given in Table 1.

Table 1: Formulation of 2PP structuring sample

\begin{tabular}{cc}
\hline Component & $\begin{array}{c}\text { Fraction } \\
\text { (weight\%) }\end{array}$ \\
\hline BYK E 410 & 1.5 \\
TTA with 0.1\% B3FL & 20 \\
Darocur 1173 & 1 \\
TEGO RAD 2200N & 77.5 \\
\hline
\end{tabular}

The formulation was then degassed under high vacuum until no bubbles were visible any more. Subsequently, a $200 \mu \mathrm{m}$ film was draw casted within a cavity formed by an object slide and adhesive foil and left for a few hours to obtain thixotropicity.<smiles>C=CC(=O)OCC(CC)(COC(=O)C=C)COC(=O)C=C</smiles>

TTA<smiles>CCCCC(=O)c1ccc(C#Cc2ccc3c(c2)C(=O)c2cc(C#Cc4ccc(N(Cc5ccccc5)C(=O)Cc5ccccc5)cc4)ccc2-3)cc1</smiles>

Fig. 3: Chemical structure of the monomer TTA and the two-photon-initiator B3FL.

\subsection{DLW and Post-Processing}

For our structuring experiments we used an ultra fast ti$\tan$ - sapphire laser system (Mai Tai, pulse duration: $120 \mathrm{fs,}$ repetition rate: $1 \mathrm{kHz}$ ) as described earlier.[10] After DLW, the film was cured in an inert gas atmosphere for $5 \mathrm{~min}$ at $20 \mathrm{~mW}$. 


\section{Results and Discussion}

\subsection{Matrix formulation}

Our matrix of choice had to be PDMS-based due to the remarkable optical, mechanical and thermal properties. For being able to carry out the manufacturing process as shown in Fig. 1 we needed to find a way to change the rheology of the liquid in the presence of a monomer formulation to thixotropic in a reasonable period of time. It turned out, that silicone polyether acrylate - probably due to the polyether domains - becomes thixotropic within a few hours, when the rheology additive BYK E 410 is added. The acrylate functionalities provided the matrix material with rapid curability in the final step of the manufacturing process. The fact, that acrylate groups are present in both the matrix and the monomer formulation is thought to enhance the compatibility of the corresponding photopolymers due to copolymerization.

\subsection{Monomer selection}

As our waveguides concept is based on a local refractive index increase, the first prerequisite for monomers was a higher refractive index than the matrix material. Additionally, compatibility with the matrix used was indespensable in order to ensure transparency and low optical damping behavior of the waveguide material respresenting the most difficult issue. In contrast to earlier approaches, high refractive monomer is enriched during $2 \mathrm{PP}$ in the structuring volume via diffusion, so the viscosity and diffusibility of the monomer represented a central criterion. Furthermore, the monomer formulation should be able to form a crosslinked network upon 2PP and exhibit a reasonable reactivity (2PP and UV) for fabrication speed reasons. The volatility of the latter had to be non-significant so as not to lose monomer during the fabrication process. After some screening TTA turned out to be suitable for our purpose.

\subsection{PP Fabrication of Waveguides}

Preliminary structuring tests of our material allowed for the proof of concept, that waveguiding is in fact possible: Comparatively strong phase contrasts were obtained, which should be due to the high diffusibility of the monomer in the thixotropic medium (see Fig. 2).
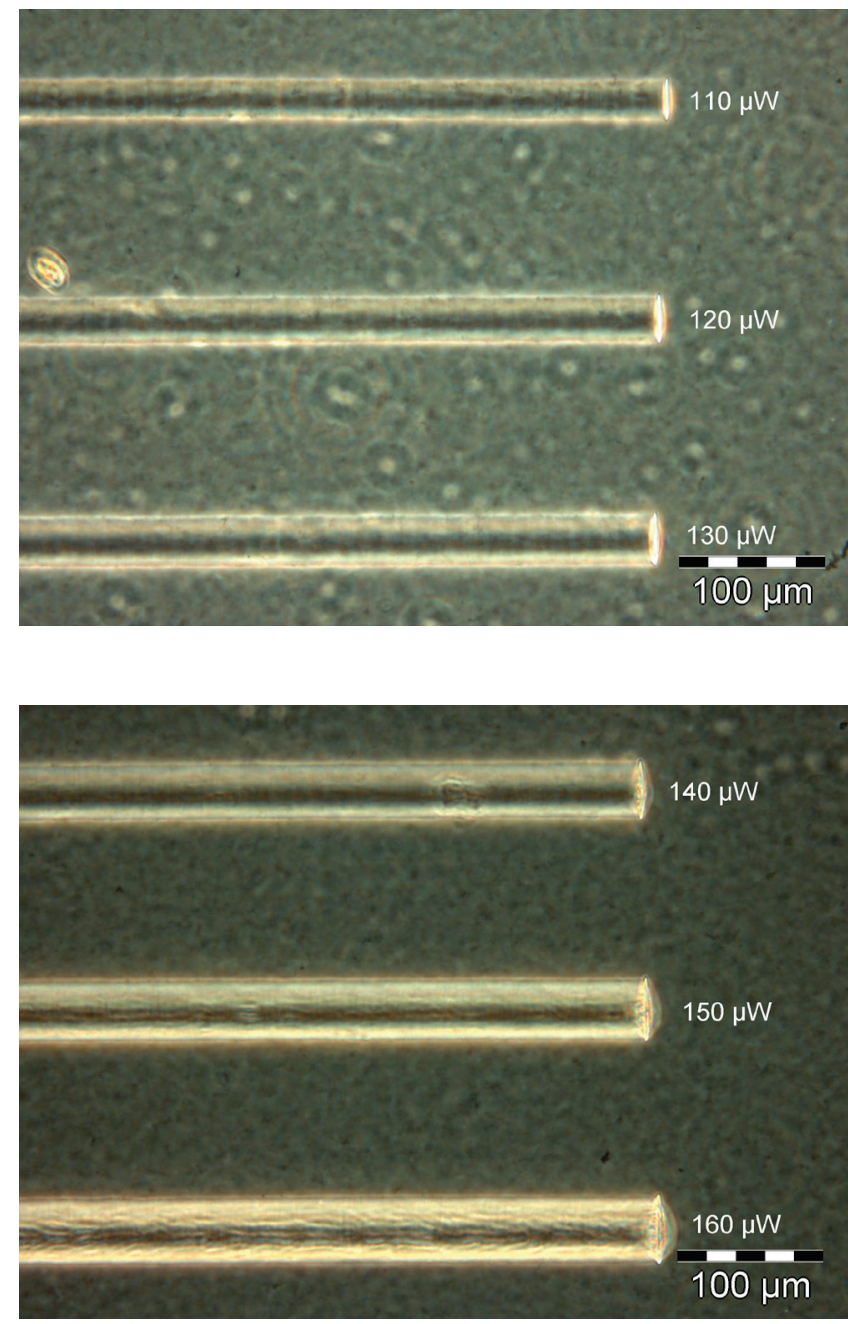

Fig. 2: Phase contrast image of waveguide structures fabricated at writing speed of $4 \mathrm{~mm} / \mathrm{min}$ and 110,120 and 130 (top) 140,150 and $160 \mu \mathrm{W}$ laser power (bottom).

Waveguides were written in depth of $120 \mu \mathrm{m}$ as a consequence of oxygen-inhibition in the surface region. The higher the laser intensity was the better phase contrasts could be achieved, which was limited by burnt regions within the waveguide at high intensities. Good waveguiding performance could be qualitatively demonstrated by preliminary coupling in experiments: A film of the material with inscribed waveguide bundles was cut and brought into the white light of a microscope giving a strongly illuminated cross-section of the bundle. 

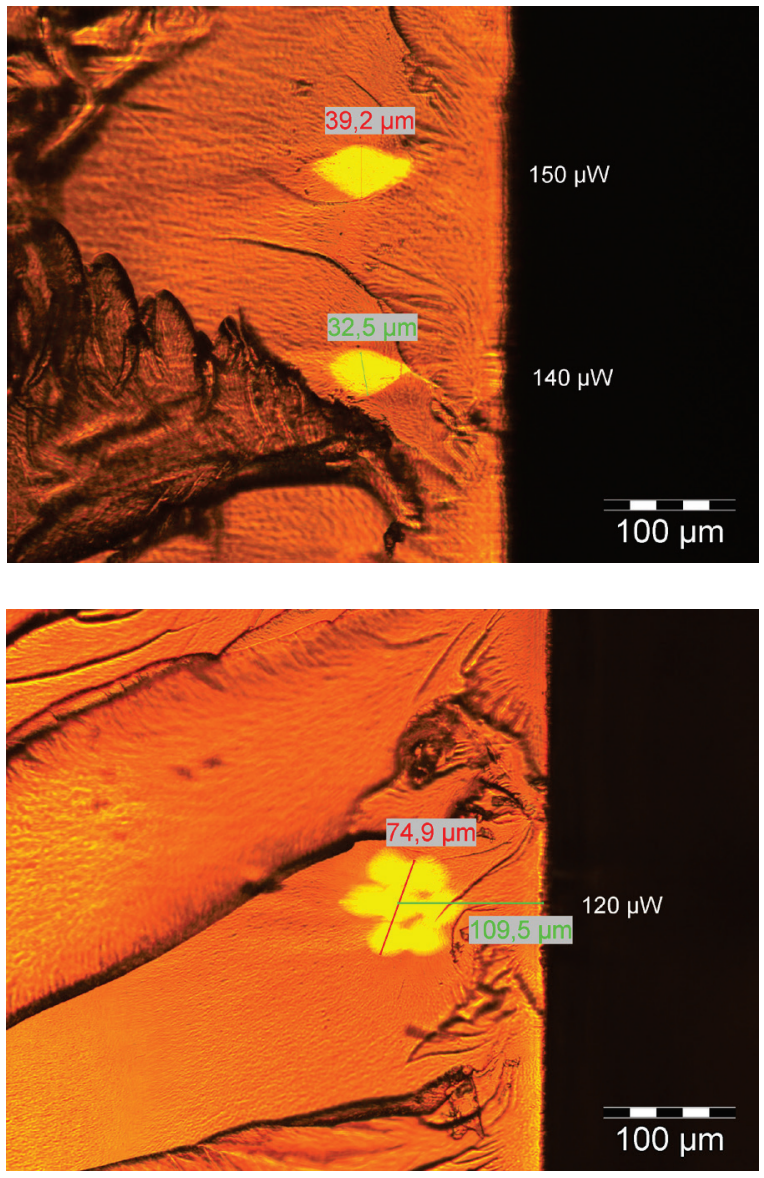

Fig. 4: Top: Cross-section of waveguides (yellow spots) written at 140 and $150 \mu \mathrm{W}$ laser power and at $4 \mathrm{~mm} / \mathrm{min}$ writing speed; dark regions represent unevenness of the cut. Bottom: Cross-section of a bundle of seven waveguides written at $120 \mu \mathrm{W}$ laser power and at $4 \mathrm{~mm} / \mathrm{min}$ writing speed; red line: bundle diameter, green line: depth of middle waveguide.

Fig. 4 also shows that the waveguide did not significantly sink towards the object slide after the structuring and UVcuring, which is an important aspect for printed circuit board applications.

\section{Conclusion}

In summary, we were able to show qualitatively that it is possible to create efficient waveguides via $2 \mathrm{PP}$ in a thixotropic medium of hydrophilic polysiloxane and photoreactive monomer formulation. While former manufacturing techniques exhibited time consuming steps as swelling or evaporating bulk monomer, the processing of our material is very simple: The draw-casted sample becomes thixotropic within a few hours, then waveguides can already be structured and the final step is only a short UV-curing period. The fact, that 2PP is carried out in a liquid enables enhanced diffusion of high refractive monomer into the illuminated regions compared to crosslinked networks and thus a large increase in refractive index. Currently, we are aiming to even further enhance the phase contrast and reduce shrinkage of the system presented here by addition of tetrafunctional thiol so as to obtain a partial thiol-ene polymerization with the acrylate groups.

\section{Acknowledgments}

We acknowledge the financial support by the Austrian FFG contracts 815417 and 819717.

\section{References}

[1] H. B. Sun, S. Kawata: Adv. Polym. Sci. 170 (2004) 169.

[2] K. Miura, J. Qiu, H. Inouye, I. Mitsuya, K. Hirao: Appl. Phys. Lett., 71, (1997) 3329.

[3] R. Houbertz, G. Domann, C. Cronauer, A. Schmitt, H. Martin, J. U. Park, L. Frohlich, R. Buestrich, M. Popall, U. Streppel, P. Dannberg, C. Wachter, A. Brauer: Thin Solid Films, 442, (2003) 194.

[4] M. Oubaha, R. Copperwhite, A. Gorin, C. Boothman, M. O’Sullivan, R. Gadonas, C. McDonagh, B. D. Mac Craith: Appl. Surf. Sci, 257, (2011) 2995.

[5] J. Ishihara, K. Komatsu, O. Sugihara, T. Kaino: Appl. Phys. Lett., 90, (2007) 033511/1.

[6] T. Watanabe, N. Ooba, S. Hayashida, T. Kurihara and S. Imamura: J. Lightwave Technol., 16, (1998) 1049.

[7] R. Infuehr, N. Pucher, C. Heller, H. Lichtenegger, R. Liska, V. Schmidt, L. Kuna, A. Haase and J. Stampfl: Appl. Surf. Sci., 254, (2007) 836.

[8] S. Bichler, S. Feldbacher, R. Woods, V. Satzinger, V. Schmidt, G. Jakopic, G. Langer, and W. Kern: Proc. SPIE, 7413, (2009) 74130W-1.

[9] Z. Li, M. Siklos, N. Pucher, K. Cicha, A. Ajami, W. Husinsky, A. Rosspeintner, E. Vauthey, G. Gescheidt, J. Stampfl, R. Liska: J. Polym. Sci, Part A, submitted 2011.

[10] V. Schmidt, L. Kuna, V. Satzinger, R. Houbertz, G. Jakopic, G. Leising: Proc. SPIE, 6476, (2007), 64760P

(Received: May 31, 2011, Accepted: October 26, 2011) 